\title{
ON THE STRUCTURE OF NONSTANDARD MODELS OF ARITHMETIC
}

\author{
R. G. PHILLIPS
}

Abstract. In this paper we show that the additive group of each nonstandard model ${ }^{*} Z$ of the integers $Z$ is isomorphic to the group $\langle F \times Z,+\rangle$ where $F$ is a direct sum of $\alpha$-copies of the rationals $Q, \alpha$ the cardinality of ${ }^{*} Z$, and + is defined by: $(a, x)$ $+(b, y)=(a+b, x+y+g(a, b))$ for certain functions $g$ mapping from $F \times F$ to $Z$.

Introduction. Let ${ }^{*} Z$ denote any nonstandard model of arithmetic and set

$$
{ }^{*} Z / Z=\left\{\bar{a}: a \in{ }^{*} Z\right\}, \quad \bar{a}=a+Z .
$$

For each ${ }^{*} Z$ we let $K=K\left({ }^{*} Z\right)$ denote the set of elements of $* Z$ of infinite height in $* Z$. (An element has infinite height if it is divisible by every positive integer.) It is easy to see that $\bar{a} \cap K$ never contains more than one element. Kemeny [2] asked whether there existed a $* Z$ so that $\bar{a} \cap K$ contained exactly one element for every $a \in \in^{*} Z$. The importance of Kemeny's question lies in the fact that if such a ${ }^{*} Z$ existed, Goldbach's conjecture could be proven false; however, it was shown by Gandy [1] and Mendelson [3] that this question is answered in the negative.

The purpose of this paper is to analyze, in more detail, the structure of the models $* Z$, based on ideas suggested by Kemeny's and Mendelson's work and by the work of MacDowell and Specker [6]. The basic results about nonstandard models used in this paper can be found in Robinson [5]. We begin with Mendelson's solution to Kemeny's problem.

\section{The additive group of $* Z$.}

TheOREM 1 (Mendelson). Any nonzero ring homomorphism of $* Z$ into itself is an order-preserving isomorphism.

Presented to the Society, August 28, 1969 under the title $A$ canonical form for the additive group of nonstandard models of arithmetic; received by the editors February 16, 1970.

AMS 1970 subject classifications. Primary 02H05, 02H15, 02H20; Secondary 02HXX.

Key words and phrases. Nonstandard models, additive groups, nonstandard models of arithmetic, Goldbach's conjecture. 
Now suppose $K \cap \bar{a}$ consists of exactly one element for each $a$ in some ${ }^{*} Z$. Then, clearly, the additive group of ${ }^{*} Z$ may be written as $K \oplus Z$. Hence, the map $f(x+n)=n$, where $x \in K$ and $n \in Z$, is not an order-preserving isomorphism, in contradiction to Theorem 1.

That the set $K$ plays an important role in determining the structure of ${ }^{*} Z$ is seen in the work of MacDowell and Specker. There, the additive group of ${ }^{*} Z$ is shown to be isomorphic to $K \oplus J$ where $J$ is some subgroup of the product group $\prod_{p=1}^{\infty} Z_{p}, Z_{p}$ being the integers $\bmod p$.

We now present a canonical form (Phillips [4]) for the additive group of each ${ }^{*} Z$ in which, essentially, only the addition varies from model to model. Given ${ }^{*} Z$, let $h:{ }^{*} Z / Z \rightarrow^{*} Z$ be a choice function; that is, $h(\bar{a}) \in \bar{a}$. We "normalize" $h$ so that $h(\overline{0})=0$. Then the map $\alpha_{h}$ given by

$$
a \rightarrow(\bar{a}, a-h(\bar{a}))
$$

is a one-to-one map of ${ }^{*} Z$ on to $* Z / Z \times Z$.

THEOREM 2. Let $* Z / Z h Z$ denote the group whose elements are $* Z / Z \times Z$ and whose group operation is defined to be

$$
(\bar{a}, x)+(\bar{b}, y)=(\overline{a+b}, x+y+h(\bar{a})+h(\bar{b})-h(\overline{a+b})) .
$$

Then $\alpha_{h}$ is an additive isomorphism of $* Z$ onto $* Z / Z h Z$.

Proof. We have

$$
a+b \rightarrow(\overline{a+b}, a+b-h(\overline{a+b}))
$$

and hence we may define addition as

$$
(\bar{a}, a-h(\bar{a}))+(\bar{b}, b-h(\bar{b}))=(\overline{a+b}, a+b-h(\overline{a+b})) .
$$

It is easy to see that this addition is equivalent to that defined by (i) and the theorem follows.

Definition 1. Let $F$ be some direct sum of infinitely many copies of the rationals $Q$. By $F_{0}$ we mean the set of all functions $g: F \times F \rightarrow Z$ such that

1. $g(a, 0)=0$,

2. $g(a, b)=g(b, a)$,

3. $g(a, b)+g(a+b, c)=g(a, b+c)+g(b, c)$.

If $g \in F_{0}$, we let $F g Z$ denote the set $F \times Z$ together with the additive operation defined as

$$
(a, x)+(b, y)=(a+b, x+y+g(a, b)) .
$$


The next theorem is immediate.

THEOREM 3. For each $g \in F_{0}, F g Z$ is an abelian group.

THEOREM 4. For each ${ }^{*} Z$ of cardinal $\alpha,{ }^{*} Z / Z$ is isomorphic to a direct sum of $\alpha$ copies of the rationals $Q$, which in turn is isomorphic to $K\left({ }^{*} Z\right)$.

Proof. See MacDowell-Specker [6].

THEOREM 5. For each $* Z$ of cardinal $\alpha$ there exists a function $g \in F_{0}$, where $F$ is a direct sum of $\alpha$ copies of $Q$, so that the additive group of ${ }^{*} Z$ is isomorphic to $\mathrm{FgZ}$; the isomorphism mapping $n \in Z$ onto $(0, n)$ in $\mathrm{FgZ}$.

Proof. Let $\pi: F \rightarrow^{*} Z / Z$ be the isomorphism given by Theorem 4 and let

$$
g(a, b)=h(\pi(a))+h(\pi(b))-h(\pi(a)+\pi(b)),
$$

where $h:{ }^{*} Z / Z \rightarrow^{*} Z$ is a choice function, $h(\overline{0})=0$. Then $F g Z$ is isomorphic to ${ }^{*} Z / Z h Z$ by the map $(a, x) \rightarrow(\pi(a), x)$ and now the theorem follows from Theorem 2.

3. The set $F_{1}$. Let $F$ be an infinite direct sum of $\alpha$ copies of $Q$ and let $F_{1}=F_{1}(\alpha)$ denote the set of all maps $g \in F_{0}$ such that for some nonstandard model ${ }^{*} Z$ of cardinal $\alpha$, the additive group of ${ }^{*} Z$ is isomorphic to $\mathrm{Fg} Z$ as in Theorem 5 .

THEOREM 6. $g \equiv 0$ is not in $F_{1}$.

Proof. Follows from Theorem 1. Hence, there are no linear choice functions from ${ }^{*} Z / Z \rightarrow^{*} Z$.

Since ${ }^{*} Z / Z$ and $F$ are isomorphic, we now agree to identify corresponding elements in both structures.

ThEOREM 7. There exists $a g \in F_{1}$ such that $g(a, b)=0$ for each $a$, $b \in F$ where $a \cap K \neq \varnothing$ and $b \cap K \neq \varnothing$. Hence $K \oplus Z$ is an additive subgroup of * $Z$.

Proof. If $x \in a \cap K$, set $h(a)=x$; define the choice function $h$ arbitrarily for the remaining $a \in F$. Then $h$ is linear on the set of $a$ where $a \cap K \neq \varnothing$.

Let $a=\left(a_{1}, a_{2}, \cdots\right)$ and $b=\left(b_{1}, b_{2}, \cdots\right)$. We set $e(a, b)$ equal to the finite cardinal of the set

$$
E=(a, b)=\left\{i \text { : neither } a_{i} \text { nor } b_{i} \text { are integers or } a_{i} \neq-b_{i}\right\} .
$$

THEOREM 8. There exists a $g \in F_{1}$ such that $0 \leqq|g(a, b)| \leqq e(a, b)$ for all $a, b \in F$. 
Proof. Let $A=\left\{x_{1}, \cdots, x_{i}, \cdots\right\}$ be a basis for $* Z / Z$ over $Q$ and we identify $r_{i} x_{i}+\cdots+r_{j} x_{j}$ in $* Z / Z$ with $a \in F$ where $a_{p}=0$ if $p<i$ or $p>j$ and $a_{p}=r_{p}$ if $i \leqq p \leqq j$. We define $h\left(x_{i}\right) \in x_{i}$ arbitrarily and if $a=a_{i} x_{i}+\cdots+a_{j} x_{j}$ we set

$$
h(a)=\sum_{p=i}^{j}\left[a_{p} h\left(x_{p}\right)\right]
$$

where $[x]$ denotes the greatest integer function if $x \geqq 0$ and $[x]$ $=-[-x]$ if $x<0\left([x]\right.$ can be defined by first embedding ${ }^{*} Z$ in $\left.{ }^{*} Q\right) . h$ can be shown to be a choice function on ${ }^{*} Z / Z \rightarrow^{*} Z$ and hence we set

$$
g(a, b)=h(a)+h(b)-h(a+b) .
$$

Thus

$$
g(a, b)=\sum_{i \in E(a, b)}\left(\left[a_{i} h\left(x_{i}\right)\right]+\left[b_{i} h\left(x_{i}\right)\right]-\left[\left(a_{i}+b_{i}\right) h\left(x_{i}\right)\right]\right),
$$

using familiar properties of $[x]$. The theorem now follows from the fact that for all $x$ and $y$,

$$
[x]+[y]-[x+y]=\left\{\begin{array}{r}
1 \\
-1
\end{array}\right.
$$

One might ask if instead of using choice functions from $F \rightarrow^{*} Z$, could we define $g$ with simpler functions. The next theorem gives a partial answer to this question:

Theorem 9. If $h: F \rightarrow Z$ and $g(a, b)=h(a)+h(b)-h(a+b)$, then $g \in F_{0}-F_{1}$.

Proof. It is not hard to show that in $F g Z$ we have

$$
(0, n) \cdot\left(a / n, x_{n}\right)=\left(a, n x_{n}+n h(a / n)-h(a)\right) .
$$

Hence, for each $n$ let $x_{n}=-h(a / n)$. Then

$$
(0, n) \cdot\left(a / n, x_{n}\right)=(a,-h(a))
$$

for each $n$ and each $a$. Thus each element $(a,-h(a))$ has infinite height in $F g Z$ which implies $K \cap \bar{a} \neq \varnothing$ for each $a \in^{*} Z$ if the additive group of $* Z$ were isomorphic to $F g Z$.

\section{BIBLIOGRAPHY}

1. R. O. Gandy, A note on a paper of Kemeny's, Math. Ann. 136 (1958), 466. MR $20 \# 5141$.

2. J. G. Kemeny, Undecidable problems of elementary number theory, Math. Ann. 135 (1958), 160-169. MR 20 \#5140. 
3. E. Mendelson, On non-standard models for number theory, Essays on the Foundation of Mathematics, Magnes Press, Hebrew University, Jerusalem, 1961, pp. 259268. MR 29 \#1141.

4. R. G. Phillips, $A$ canonical form for the additive group of non-standard models of arithmetic, Notices Amer. Math. Soc. 16 (1969), 795. Abstract \#667-133.

5. A. Robinson, Non-standard analysis, North-Holland, Amsterdam, 1966. MR 34 \#5680.

6. R. MacDowell and E. Specker, Modelle der Arithmetik, Proc. Sympos. Infinitistic Methods, Foundations of Mathematics (Warsaw, 1959), Warsaw, 1961, pp. 257-263.

University of South Carolina, Columbia, South Carolina 29208 